\title{
A monitor for neutron flux measurements up to 20 $\mathrm{MeV}$
}

\author{
A. Öhrn ${ }^{1}$, J. Blomgren ${ }^{1}$, H. Park ${ }^{2}$, S. Khurana ${ }^{3}$, R. Nolte ${ }^{3}$, D. Schmidt ${ }^{3}$, and K. \\ Wilhelmsen ${ }^{4}$ \\ ${ }^{1}$ Department of Neutron Research, Uppsala University, Sweden \\ ${ }^{2}$ Korean Research Institute of Standards and Science, Daejeon, Korea \\ ${ }^{3}$ Physikalisch-Technische Bundesanstalt, Braunschweig, Germany \\ ${ }^{4}$ Swedish Defense Research Agency, Stockholm, Sweden \\ E-mail: angelica.ohrn@tsl.uu.se
}

\begin{abstract}
A liquid scintillation detector aimed for neutron energy and fluence measurements in the energy region below $20 \mathrm{MeV}$ has been calibrated using monoenergetic and white spectrum neutron fields. Careful measurements of the proton light output function and the response matrix have been performed allowing for the application of unfolding techniques using existing codes. The response matrix is used to characterize monoenergetic neutron fields produced by the $T(d, n)$ at a lowenergy deuteron accelerator installed at the Swedish Defense Research Agency (FOI).
\end{abstract}




\section{Introduction}

The strongly expanding importance of fast neutrons in a number of applications requires steps to be taken to improve the technology for neutron fluence and energy measurements in various energy ranges. This requirement was addressed and the current situation summarized at a recent workshop, the International Workshop on Neutron Field Spectroscopy in Science held in Pisa, Italy, June 4 - 8, 2000 [1].

For the application considered in this work - a monitor for fluence and energy measurements in the energy region from a few $\mathrm{MeV}$ to about $20 \mathrm{MeV}$ - there are several options possible, but if resolution and detection efficiency are taken into consideration, the most attractive alternatives seem to be the organic scintillator with or without applying time-of-flight techniques. In the present application the neutron source is continuous and therefore the time-of-flight technique is out of question. The obvious choice is therefore to perform pulse-height spectrum measurements and to apply unfolding techniques.

In the present paper a calibration procedure for a liquid scintillator with pulse-shape discrimination possibilities is described. The method is based on the procedures developed at the PhysikalischTechnische Bundesanstalt (PTB), Braunschweig, Germany [2] and involves measure-ments of pulseheight spectra and unfolding of these spectra with existing computer codes using carefully recorded response functions at several energies in the region of interest. The procedure presented in this paper goes beyond the previously applied methods in that both the experimental and calculated response matrices are used with existing unfolding codes to determine the energy and fluence of monoenergetic neutron fields and make comparisons with time-of-flight (TOF) methods.

\section{Experimental methods}

For neutron energies below $20 \mathrm{MeV}$, the response matrix of a scintillation detector can be calculated using Monte Carlo codes provided the specific light outputs for protons, deuterons and alpha particles are known for that particular detector. For energies above about $8 \mathrm{MeV}$, however, no available Monte Carlo code is capable of describing the response of a scintillation detector in full detail, because the required sufficiently detailed multi-differential emission cross sections for alpha particles from the ${ }^{12} \mathrm{C}\left(\mathrm{n}, \mathrm{n}{ }^{\prime} 3 \alpha\right)$ reaction are not available. Hence, the characterization of scintillation detectors always requires an experimental investigation of the detector response. The standard procedure developed at PTB for the characterization of scintillation detectors uses monoenergetic and breakup neutrons produced with the $\mathrm{D}(\mathrm{d}, \mathrm{n})$ reaction.

The standard procedure is satisfactory for the application of the TOF method. For the application of unfolding techniques, however, a proper description of the full response matrix is required since any deviation of the response matrix from the 'true' pulse-height response of the detector would cause spurious structures in the unfolded spectral fluence.

For this purpose, a method described by Dekempeneer et al. [3] has been adopted. A white neutron beam measurement is used to obtain a smooth light output function for protons and an experimental response matrix with sufficient resolution in neutron energy. This method has been tested on a liquid scintillator to be used as a neutron monitor for a DT neutron generator, i. e., a commercial cylindrical detector cell of the MAB-1F type filled with BC501 scintillator liquid.

The PTB standard procedure for the determination of the relevant properties of an organic scintillation detector has been described in detail elsewhere [2]. Only the results obtained for the particular detector under study are summarized here.

Five neutron beams were produced by deuterons with energies of 5.01, 7.12, 9.06, 10.30, and 11.27 $\mathrm{MeV}$ using a deuterium gas target at the PTB neutron scattering facility. The energies of the monoenergetic neutrons were $7.95,10.05,11.93,13.12$, and $14.05 \mathrm{MeV}$. The maximum energy of the corresponding breakup continua was about $6.5 \mathrm{MeV}$ below that of the monoenergetic neutrons. About 30 narrow TOF windows were placed on the monoenergetic neutrons and the breakup continuum to produce pulse-height spectra which were used to determine the proton light output and the efficiency of the detector.

The Monte Carlo code NRESP7 [4] was used to calculate pulse-height spectra for comparison with experimental spectra obtained at the five energies where monoenergetic neutrons from the $D(d, n)^{3} \mathrm{He}$ 
reaction were available. By fitting these calculated spectra to the experimental ones, the light output function for protons, i.e., the pulse height corresponding to the recoil proton edge, was determined with an iterative procedure. The pulse height was measured using a calibration with photon sources.

The fluences of the monoenergetic neutrons were measured with the PTB 4"x1" NE213 reference detector. This detector was repeatedly compared with the PTB recoil proton telescope. For a selected pulse-height threshold, the efficiency of the detector is known with an uncertainty of about $1.5 \%$ [5]. The mean ratio of the fluence determined with the present BC501 detector and that measured with the PTB reference detector was $1.018+/-0.009$, which is within the range of results for other detectors [2].

A white neutron beam was produced at the PTB time-of-flight (TOF) facility by bombarding a thick Be target with a $19 \mathrm{MeV}$ proton beam from the PTB isochronous cyclotron. The maximum energy of the neutron field at an emission angle of $0^{\circ}$ is $17.15 \mathrm{MeV}$. The neutron field was collimated by one of the collimators of the PTB TOF facility. The scintillation detector was positioned at a distance of $27.39 \mathrm{~m}$ from the Be target.

Energy calibration of the pulse-height spectra was established using ${ }^{137} \mathrm{Cs},{ }^{22} \mathrm{Na}$ and ${ }^{207} \mathrm{Bi}$ photon sources. The calibration of the measured pulse height in electron energies and the electronic offset were determined by fitting pulse-height spectra calculated with the PHRESP code [6] to the experimental spectra.

To establish an experimental response matrix, the PH spectra obtained with the white beam have to be normalized to unit fluence at the centre of the detector. This normalization was carried out by fitting PH spectra calculated with NRESP7 to the experimental ones. The fit was restricted to the region extending from the beginning of the flat plateau to the recoil proton edge. This region is essentially determined by np scattering and can be accurately described by NRESP7.

\section{Test of calculated and experimental response matrices in monoenergetic neutron fields}

In the present work it was considered important to test the calculated and experimental response matrices in well-defined monoenergetic neutron fields in an energy region of relevance for the actual application. Such a test was regarded as particularly relevant, because of the observed deviations between the experimentally determined response matrix and the calculated one.

The experimental response matrix was tested in monoenergetic neutron fields with energies between $14 \mathrm{MeV}$ and $15.5 \mathrm{MeV}$. These fields were produced by the $\mathrm{T}(\mathrm{d}, \mathrm{n})^{4} \mathrm{He}$ reaction. Deuteron beams of 242, 412 and $643 \mathrm{keV}$ were produced with the PTB $3.5 \mathrm{MeV}$ van-de-Graaff accelerator. The spectral distributions of the neutron fields were calculated with the TARGET code [7]. The calculated average energies at $0^{\circ}$ were $14.85,14.99$ and $15.60 \mathrm{MeV}$, respectively, and the corresponding calculated FWHM of the peaks amounted to 451, 699 and $644 \mathrm{keV}$. For the $412 \mathrm{keV}$ deuteron beam, measurements were also carried out at a neutron emission angle of $98^{\circ}$. At this angle, the $\mathrm{T}(\mathrm{d}, \mathrm{n})^{4} \mathrm{He}$ reaction shows so-called kinematical focusing, i.e., the energy of the emitted neutrons is almost independent of the energy of the incident deuterons. Hence, broadening of the spectral distribution of the neutrons due to the energy loss of the deuterons in the $\mathrm{Ti}(\mathrm{T})$ layer is very small. In this particular case, the neutron field had a peak energy of $13.98 \mathrm{MeV}$ and a FWHM of only $17 \mathrm{keV}$ according to the TARGET calculations which were carried out neglecting the angular straggling of the deuterons in the target.

The pulse-height spectra obtained during the present measurements were unfolded with the MAXED code [8] which is part of the UMG code package [9]. It was known from the TOF measurements that the spectral neutron distribution showed a $\mathrm{D}(\mathrm{d}, \mathrm{n})$ background peak between $2 \mathrm{MeV}$ and $4 \mathrm{MeV}$ in addition to the dominant $\mathrm{T}(\mathrm{d}, \mathrm{n})$ peak at energies above $14 \mathrm{MeV}$. Using this preinformation, the unfolding was carried out in two steps. First, a high $\mathrm{PH}$ threshold of $7 \mathrm{MeV}$ corresponding to a neutron energy of a about $11.2 \mathrm{MeV}$ was used to select those events which could not be caused by the low-energy background. The spectral fluence distribution obtained from this restricted unfolding exhibited a prominent peak and some background at intermediate energies. Second, this peak was used as preinformation for the next step of the unfolding procedure that comprised the pulse-height spectrum above a PH threshold of $280 \mathrm{keV}$. 


\section{Results and discussion}

The experimental and calculated response matrices were applied with unfolding codes available in the literature to determine the energy and fluence of monoenergetic neutron fields. Comparison with results from TOF measurements was performed.

Determination of the neutron energy has been performed from experiments performed with neutron energies of 13.98, 14.85 and $15.60 \mathrm{MeV}$. The pulse-height spectrum has been unfolded using MAXED as well as GRAVEL, the latter being another unfolding code in the UMG package [10]. Both codes give a good description of the neutron distribution. However, some spurious structures are generated especially in the low-energy region. A small structure around $11 \mathrm{MeV}$ can also be seen (see Fig. 1). There is no significant difference between GRAVEL and MAXED when determining the neutron energy (see Table 1). The result from unfolding with the experimental response matrix is in better agreement with the result from the TOF measurements than the result from the calculated response matrix.
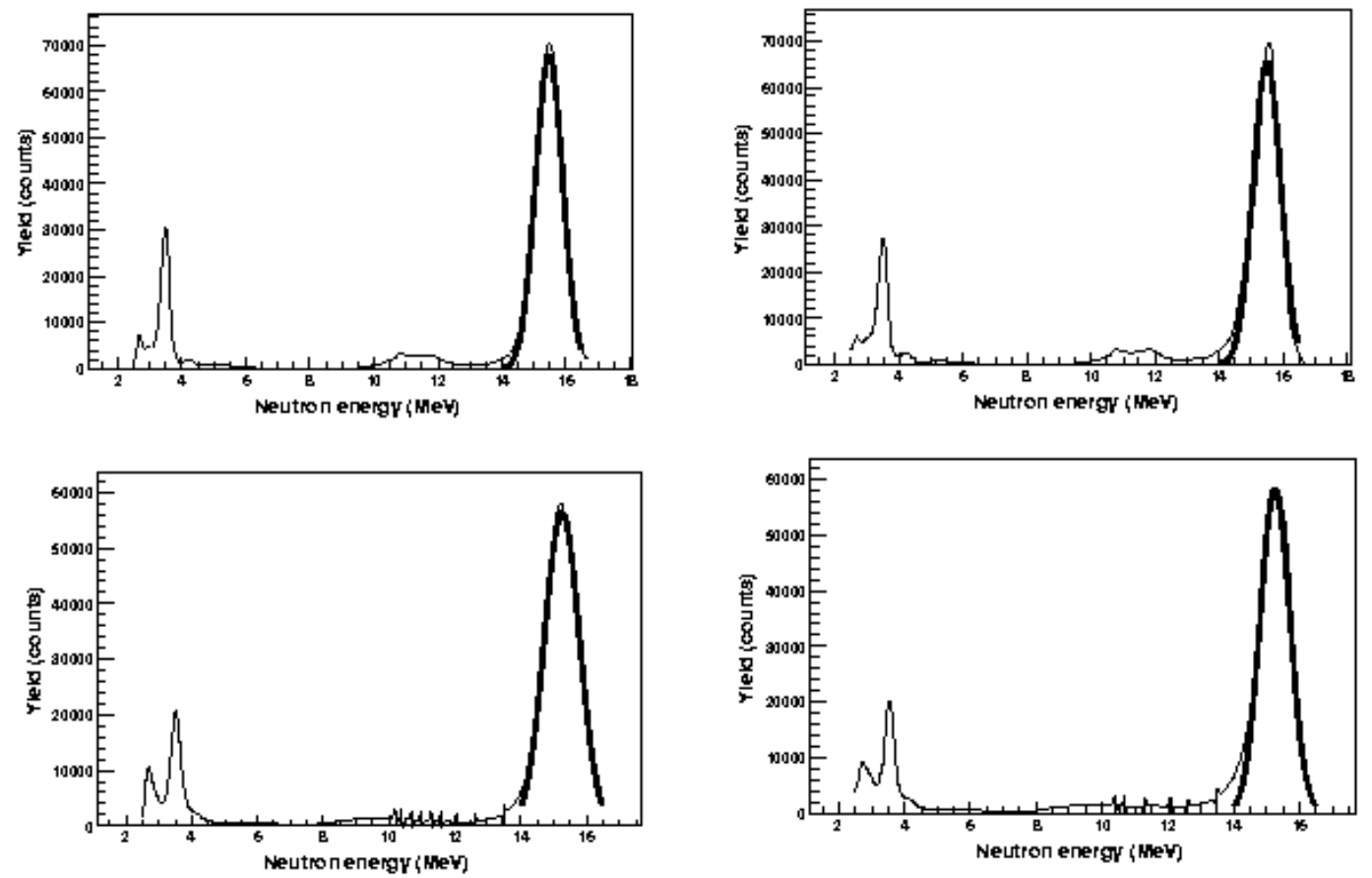

Fig. 1. Results from the 15.60 MeV measurements. The unfolding in the left panels are performed with GRAVEL and those in the right panels with MAXED. In the upper panels the experimental response is used and in the lower panels the calculated one.

The absolute efficiency of the detector has been determined using the PTB proton recoil telescope. These measurements have verified that the efficiency, in terms of total number of counts in the detector above a threshold set at a low pulse height, agrees with what can be expected with the used tagged neutron flux. This has been corroborated using the fact that the pulse height spectrum in the high-energy end is dominated by events due to np scattering, because the np scattering cross section is well known at these energies.

The neutron fluence has been determined by integrating the $T(d, n)$ peak from $E_{0}-\Gamma$ to $E_{0}+\Gamma$, where $\mathrm{E}_{0}$ is the centroid and $\Gamma$ is the FWHM (see Fig. 1). The intrinsic uncertainty of this method has been determined to $0.5 \%$ on the average with a worst case of $1.0 \%$. 
Table 1. Results of the neutron energy measurements. Errors are statistical only.

$E_{n}=13.98 \mathrm{MeV}$

\begin{tabular}{|l|l|l|}
\hline Unfolding code & Response matrix & Neutron energy $(\mathrm{MeV})$ \\
\hline GRAVEL & Experimental & $13.91+/-0.02$ \\
\hline GRAVEL & Calculated & $13.67+/-0.01$ \\
\hline MAXED & Experimental & $13.92+/-0.03$ \\
\hline MAXED & Calculated & $13.70+/-0.02$ \\
\hline
\end{tabular}

$E_{n}=14.85 \mathrm{MeV}$

\begin{tabular}{|l|l|l|}
\hline GRAVEL & Experimental & $14.69+/-0.02$ \\
\hline GRAVEL & Calculated & $14.43+/-0.01$ \\
\hline MAXED & Experimental & $14.71+/-0.03$ \\
\hline MAXED & Calculated & $14.47+/-0.02$ \\
\hline
\end{tabular}

$E_{n}=15.60 \mathrm{MeV}$

\begin{tabular}{|l|l|l|}
\hline GRAVEL & Experimental & $15.48+/-0.01$ \\
\hline GRAVEL & Calculated & $15.26+/-0.01$ \\
\hline MAXED & Experimental & $15.51+/-0.03$ \\
\hline MAXED & Calculated & $15.26+/-0.01$ \\
\hline
\end{tabular}

The fluence obtained from the time-of-flight spectrum from the $15.60 \mathrm{MeV}$ measurement agrees well with the fluence derived from unfolding with the experimental response matrix. The deviations are $0.7 \%$ (GRAVEL) and $1.9 \%$ (MAXED). The deviations are significantly larger in the unfolding with the calculated response matrix, $1.6 \%$ (GRAVEL) and $5.8 \%$ (MAXED). In all cases, the fluence obtained from unfolding is lower than that from the TOF information. Based on this information, it is concluded that the fluence can be determined with an uncertainty of $2 \%$ using this method.

\section{Conclusions}

The present work has shown that measurements in white neutrons beams can provide additional information for the specification of scintillation detectors which cannot be obtained with monoenergetic neutron beams alone. In particular, smoother experimental light outputs can be obtained and the deficiencies of the present Monte Carlo codes used for the calculation of response matrices can be circumvented. On the other hand, the application of the TOF method for the determination of experimental response matrices with white neutron beams requires very careful experimental work to avoid artefacts like those observed in the present data for neutron energies above $12 \mathrm{MeV}$. The application of the unfolding technique with experimentally determined response matrices provides a possibility for spectrometry in neutron beams over a large energy range. The present work has shown that even quite small spectral details can be resolved in the presence of other dominant structures.

\section{Acknowledgements}

The authors are indebted to A. Zimbal for guiding them through the technical pitfalls of the unfolding codes and for numerous discussions on this subject. The support of K. Tittelmeier, A. Toll and the staff of the PTB accelerator facility is gratefully acknowledged. 


\section{References}

[1] Proc. of the Int. Workshop on Neutron Field Spectrometry in Science, Technology and Radiation Protection, Pisa, Italy, June 4--8, 2000. Ed.: H. Klein, D. Thomas, H.G. Menzel, G. Curzio, and F. d'Errico, Nucl. Instr. Meth. Phys. Res. A 476, Issues 1-2, 1 (2002).

[2] D. Schmidt, B. Asselineau, R. B।"ottger, H. Klein, L. Lebreton, S. Neumann, R. Nolte, and G. Pichenot, Nucl. Instr. Meth. Phys. Res. A 476, 186 (2002).

[3] E. Dekempeneer, H. Liskien, L. Mewissen, and F. Poortmans, Nucl. Instr. Meth. Phys. Res. A 256, 489 (1987)

[4] G. Dietze and H. Klein, "NRESP4 and NEFF4: Monte Carlo Codes for the Calculation of Neutron Response Functions and Detection Efficiencies for the NE213 ScintillationDetectors", PTB Report PTB-ND-22, Physikalisch-Technische Bundesanstalt Braunschweig,1982, (and an informal supplement describing the changes of version 7, 1991).

[5] D. Schmidt and H. Klein, Precise Time-of-Flight Spectrometry of Fast Neutrons - Principles, Methods and Results. PTB Report PTB-N-35, Physikalisch-Technische Bundesanstalt Braunschweig, 1998.

[6] T. Novotny, "Photon Spectrometry in Mixed Neutron- Photon Fields using NE213 Liquid Scintillation Detectors", PTB Report PTB-N-28, Physikalisch-Technische Bundesanstalt Braunschweig, 1997.

[7] D. Schlegel, "TARGET User's Manual", PTB Laboratory Report PTB-6.41-98-1, PhysikalischTechnische Bundesanstalt Braunschweig, 1998.

[8] M. Reginatto, P. Goldhagen, and S. Neumann, Nucl. Instr. Meth. Phys. Res. A 476, 242 (1989).

[9] M. Reginatto, B. Wiegel, and A. Zimbal, UMG-Code Package, available from the Nuclear Energy Agency (NEA) Data Bank, http://www.nea.fr. 